\title{
VIII. Investigation of formulæ for the summation of certain classes of infinite series
}

\section{J.R. Young}

To cite this article: J.R. Young (1837) VIII. Investigation of formulæ for the summation of certain classes of infinite series, Philosophical Magazine Series 3, 11:64-65, 41-44, DOI: $10.1080 / 14786443708649226$

To link to this article: http://dx.doi.org/10.1080/14786443708649226

册Published online: 17 Jun 2009.

Submit your article to this journal

Џ Article views: 4

Q View related articles $₫$ 
VIII. Investigation of Formula for the Summation of certain Classes of Infinite Series. By J. R. Young, Professor of Mathematics in Belfast College.

[Continued from vol. x. p. 124, and concluded.]

LET us take $A$ of the form

$$
\frac{1}{n^{\alpha}(n+p)(n+2 p) \ldots(n+m p)},
$$

then, by the general relation referred to, we may substitute for it the expression

$$
\begin{gathered}
\frac{1}{m p}\left\{\frac{1}{n^{x}(n+p)(n+2 p) \ldots[n+(m-1) p]}\right. \\
\left.-\frac{1}{n^{x-1}(n+p)(n+2 p) \ldots(n+m p)}\right\} .
\end{gathered}
$$

Now, by the same relation, we may for the first expression within the brackets substitute

$$
\begin{aligned}
\frac{1 .}{(m-1) p} & \left\{n ^ { x } ( n + p ) ( n + 2 p ) \ldots \left[\frac{1}{[n+(m-2) p]}\right.\right. \\
& \left.-\frac{1}{n^{x-1}(n+p)(n+2 p) \ldots[n+(m-1) p]}\right\}
\end{aligned}
$$

and for the first of these we may make a like substitution; so that by proceeding in this manner we shall at length arrive at

$$
\frac{1}{2 p}\left\{\frac{1}{n^{x}(n+p)}-\frac{1}{n^{x-1}(n+p)(n+2 p)}\right\}
$$

and finally at

$$
\frac{1}{p}\left\{\frac{1}{n^{x}}-\frac{1}{n^{x-1}(n+p)}\right\}
$$

whence a rule may be easily deduced for the summation of a series of the proposed form, provided certain subordinate series can be summed. As an illustration of this suppose $x=2$, and put for abridgement

$$
\begin{aligned}
& \frac{1}{n^{2}}+\frac{1}{(n+p)^{2}}+\frac{1}{(n+2 p)^{8}}+\& \mathrm{c} .=s \\
& \frac{1}{n(n+p)}+\frac{1}{(n+p)(n+2 p)}+\frac{1}{(n+2 p)(n+3 p)}+\& c_{.}=\mathrm{S}_{1} \\
& \frac{1}{n(n+p)(n+2 p)}+\frac{1}{(n+p)(n+2 p)(n+3 p)}+\& \mathrm{c} .=\mathrm{S}_{2}
\end{aligned}
$$

Third Series. Vol. 11. No. 64. July 1837. 
$\frac{1}{n(n+p) \ldots(n+m p)}+\frac{1}{(n+p)[n+(m+1) p]}+8 \mathbf{c} .=\mathrm{S}_{m}$,

then we have the following rule for the summation of the infnite series:

$\frac{1}{n^{2}(n+p) \ldots . .(n+m p)}+\frac{1}{(n+p)^{2}(n+2 p) \ldots[n+(m+1) p]}+\& \mathrm{c}$.

From $s$ take $S_{1}$ and divide the result by $p$; from the quotient take $\mathrm{S}_{2}$ and divide the result by $2 p$; from the quotient take $S_{3}$ and divide the result by $3 p$; and so on, till the divisor becomes $m p$, which will furnish a quotient equal to the sum of the proposed series.

As to the values of the subtractive quantities, $S_{1}, S_{2}, S_{3}$, \&c., they are at once obtained from the fundamental relation with which we set ont. Thus,

$$
\begin{aligned}
& \mathrm{S}_{1}=\frac{1}{p n} \\
& \mathrm{~S}_{2}=\frac{1}{2 p n(n+p)}=\frac{\mathrm{S}_{1}}{2(n+p)} \\
& \mathrm{S}_{3}=\frac{1}{3 p n(n+p)(n+2 p)}=\frac{2 \mathrm{~S}_{2}}{3(n+2 p)} \\
& \vdots \\
& \mathrm{S}_{m}=\frac{(m-1) \mathrm{S}_{m-1}}{m[n+(m-1) p]},
\end{aligned}
$$

As an example let it be required to find the sum of the infinite series

$$
\frac{1}{1^{2} \cdot 2 \cdot 3 \cdot 4}+\frac{1}{2^{2} \cdot 3 \cdot 4 \cdot 5}+\frac{1}{3^{2} \cdot 4 \cdot 5 \cdot 6}+8 \mathrm{c} .
$$

in which $n=1, p=1$ and $m=3$.

The values of $S_{1}, S_{2}, S_{3}$, are, in this case,

$$
S_{1}=1, S_{2}=\frac{1}{4}, S_{i 3}=\frac{1}{18},
$$

hence, arranging these in a row and prefixing the subtractive sign, the operation by the rule will be as follows :

$$
\begin{aligned}
& -1 \quad-\frac{1}{4} \quad-\frac{1}{18} \\
& \frac{\pi^{8}}{6} \quad \frac{\pi^{2}}{6}-1 \quad \frac{\pi^{2}}{12}-\frac{5}{8} \quad \frac{\pi^{2}}{36}-\frac{49}{216}=\text { Sum. } \\
& \tau^{2}-1 \quad \frac{\pi^{2}}{6}-\frac{5}{4} \quad \frac{\pi^{2}}{12}-\frac{49}{72}
\end{aligned}
$$


If the series to be summed were

$$
\frac{1}{1^{2} \cdot 3 \cdot 5 \cdot 7}+\frac{1}{3^{2} \cdot 5 \cdot 7 \cdot 9}+8 c .
$$

in which $p=2$, the process would be this, viz.

$$
\begin{aligned}
& -\frac{1}{2} \quad-\frac{1}{12} \quad-\frac{1}{90} \\
& \frac{\pi^{2}}{8} \quad \frac{\pi^{2}}{16}-\frac{1}{4} \quad \frac{\pi^{2}}{64}-\frac{1}{12} \quad \frac{\pi^{2}}{384}-\frac{17}{1080}=\text { Sum. } \\
& \frac{\pi^{2}}{8}-\frac{1}{2} \quad \frac{\pi^{2}}{16}-\frac{1}{3} \quad \frac{\pi^{2}}{64}-\frac{17}{180}
\end{aligned}
$$

It would be easy, by imitating the steps of the preceding investigation, to deduce a rule for the summation of the infinite series

$$
\frac{1}{n(n+p) \ldots(n+m p)^{2}}+\frac{1}{(n+p) \ldots[n+(m+1) p]^{2}}+8 \mathrm{c} .
$$

This rule would differ from the foregoing in the following particulars, viz. instead of $S_{1}, S_{2}, S_{3}$, \&c. we shall have to employ

$$
\mathbf{S}_{1}+\frac{1}{n^{2}}, \quad \mathbf{S}_{2}+\frac{1}{n(n+p)^{2}}, \mathbf{S}_{3}+\frac{1}{n(n+p)(n+2 p)^{2}}, \text { \&c., }
$$

and these, instead of being subtracted as before from the several quantities placed under them, must themselves be diminished by those quantities.

It is clear that when $n$ and $p$ are each unity, as in the first example above, the values which here supply the place of $S_{1}, S_{2}, \& c$. will be the doubles of these quantities.

As an example, let the series

$$
\frac{1}{1 \cdot 2 \cdot 3 \cdot 4^{2}}+\frac{1}{2 \cdot 3 \cdot 4 \cdot 5^{2}}+, 8 c \text {. }
$$

be proposed, in which $n=1, p=1, m=3$,

$$
\begin{array}{cccc}
\frac{2}{6} & \frac{1}{2} & \frac{1}{9} \\
2-\frac{\pi^{2}}{6} & 2-\frac{\pi^{8}}{6} & \frac{\pi^{2}}{12}-\frac{3}{4} & \frac{31}{108}-\frac{\pi^{2}}{36}=\text { Sum. }
\end{array} .
$$


By comparing the several steps of this process with those in the working of the first example, we are led to conclude that the aggregate of two series, like this last and that of the first example, will always be accurately expressed in a finite fraction, provided the factors in the denominator be even in number, and that the difference of the two series will also be a finite fraction, if the number of these factors be odd. In all other cases the sum and difference will involve $\pi^{2}$. Thus the difference between the two series

$$
\frac{1}{1^{2} \cdot 2 \cdot 3}+\frac{1}{2^{2} \cdot 3 \cdot 4}+\frac{1}{3^{2} \cdot 4 \cdot 5}+8 \text { c. }=\frac{\pi^{2}}{12}-\frac{5}{8}
$$

and

$$
\frac{1}{1 \cdot 2 \cdot 3^{2}}+\frac{1}{2 \cdot 3 \cdot 4^{2}}+\frac{1}{3 \cdot 4 \cdot 5^{2}}+8 \mathrm{c} .=\frac{\pi^{2}}{12}-\frac{3}{4}
$$

is $\frac{1}{8}$; so that actually subtracting and dividing by 2 , we have

$$
\frac{1}{1^{2} \cdot 2 \cdot 3^{2}}+\frac{1}{2^{2} \cdot 3 \cdot 4^{2}}+\frac{1}{3^{2} \cdot 4 \cdot 5^{2}}+8 \mathrm{cc} .=\frac{1}{16}
$$

this series is therefore the square of the series

$$
\frac{1}{1.2 .3}+\frac{1}{2.3 .4}+\frac{1}{3.4 \cdot 5}+\& c \text {. }
$$

It thus appears that every series of the form

$$
\frac{1}{j^{2} \cdot 2 \cdot 3 \ldots(m-1) m^{2}}+\frac{1}{2^{2} \cdot 3 \cdot 4 \ldots m(m+1)^{2}}+8 \mathrm{c} .
$$

is accurately summable when $m$ is odd, but not when $m$ is even.

Similar remarks obviously apply to the series

$$
\frac{1}{1^{2} .3 .5 \ldots(m-2) m^{2}}+\frac{1}{3^{y} \cdot 5 \cdot 7 \ldots m(m+2)^{2}}+8 \mathbf{c} \text {., }
$$

the sum of which is a determinable fraction only when the denominator of each term consists of an odd number of factors. In other cases the sum involves $\pi^{2}$.

Although in the foregoing illustrations the several terms of each series are all comected together by the sign plus, yet a glance at the general investigation will serve to show that the several deductions hold when the terms are alternately plus aud minus. 